\title{
Performance Investigation of OFDM-FSO System under Diverse Weather Conditions of Bangladesh
}

\author{
Maliha Sultana, Agnila Barua, Jobaida Akhtar, Mohammad Istiaque Reja \\ Department of Electrical and Electronic Engineering, Chittagong University of Engineering and Technology, Bangladesh
}

\begin{tabular}{l}
\hline \hline Article Info \\
\hline Article history: \\
Received Jan 31, 2018 \\
Revised Apr 2, 2018 \\
Accepted Apr 9, 2018 \\
\hline
\end{tabular}

\section{Keyword:}

Atmospheric turbulence

Free space optics

Gamma-gamma distribution

Multiple transceiver

OFDM-FSO

\begin{abstract}
Free space optical (FSO) communication systems which are deployed for last mile access, being considered as a suitable alternative technology for optical fiber networks. It is one of the emerging technologies for broadband wireless connectivity which has also been receiving growing attention due to high data rate transmission capability with low installation cost and license free spectrum. However, the widespread use of FSO technology has been hampered by the randomly time varying characteristics of propagation path mainly due to atmospheric turbulence, sensitivity to diverse weather conditions and the nonlinear responsivity of laser diode. This paper presents the performance investigation of an OFDM-FSO system over atmospheric turbulence channels under diverse weather conditions of Bangladesh. The channel is modeled with gamma-gamma distribution using 16-QAM modulation format and $4 \times 4$ multiple transceiver FSO system. All possible challenges are imposed on the system performance such as atmospheric attenuation, turbulence, pointing error, geometric loss etc. The refractive index structure parameter and atmospheric attenuation coefficient for different weather conditions are calculated by using the data, collected from Bangladesh Meteorological Department. The acquired results can be fruitful for scheming, forecasting and assessing the OFDM-FSO system's ability to transmit wireless services over turbulent FSO links under actual conditions of Bangladesh.
\end{abstract}

Copyright $@ 2018$ Institute of Advanced Engineering and Science. All rights reserved.

\section{Corresponding Author:}

Mohammad Istiaque Reja,

Department of Electrical and Electronic Engineering,

Chittagong University of Engineering \& Technology,

Chittagong-4349, Bangladesh.

Email: istique@cuet.ac.bd

\section{INTRODUCTION}

The ever increasing bandwidth demand calls for the tremendous growth and progress in information and communication technologies. Radio frequency has been being utilized for several decades but there are a set of barriers to their further expansion due to spectrum congestion, licensing issues, interference from unlicensed bands, low bit rate and multipath fading. Therefore, the research focus is towards exploiting optics to address the challenges of increasing bandwidth demand. Although fiber networks offer huge bandwidth and core network is relying mostly on it, still challenges of deploying optical fiber is not feasible in some remote and in rural areas, thus those places lack broadband network connectivity. In this scenario, terrestrial FSO technology appears to be a promising solution for the last mile network.

Free space optical (FSO) communication has achieved significant importance as a consequence of its unique features such as huge bandwidth, license free spectrum, narrow beam divergence, easy and quick deployment ability and low power requirement [1]. Moreover, it provides high speed connection up to Gbps, which is far more beyond the alternative systems. It has increasingly attracted attention for a number of applications such as last mile access, back-haul for wireless cellular networks, metro network extensions, 
military, disaster recovery, enterprise connectivity and many more. However, despite of great potential of FSO communication, its performance is limited by the unfavorable regional climatic conditions like rain, cloud, fog, haze, pollution etc. The strength of transmitted optical signal gets attenuated significantly due to absorption and scattering. Also random fluctuations in the irradiance of the received optical laser beam caused by atmospheric turbulence may lead to serious degradation in BER performance and make the communication link infeasible [2].

Orthogonal Frequency Division Multiplexing (OFDM) system has received considerable attention due to its high data rates and immunity to frequency selective multipath fading transmission technique for wireless communications, along with high spectrum efficiency. Hence, this technique is being imposed in many new broadband communication schemes, such as WLAN, DVB-T2, and WMAN 802.16m [3]. It also allows digital data to be efficiently and reliably transmitted over a radio channel, even in multipath environments [4]. The analysis of BER performance suggested that OFDM is better than CDMA which has been incorporated in most existing $3 \mathrm{G}$ systems [5].

In this paper, we present the analysis of an OFDM-FSO link over atmospheric turbulent channel considering the impact of the diverse weather conditions of Bangladesh. Higher order modulation technique such as 16-QAM is used to utilize the bandwidth of OFDM-FSO link system efficiently. The analytical transmission error performance of OFDM-FSO system is observed over moderate atmospheric turbulence by varying bit rate and link distance under different weather conditions of Bangladesh. The atmospheric attenuation coefficient for different weather conditions and refractive index structure parameter are calculated by using the data, collected from Bangladesh Meteorological Department. Gamma-Gamma distribution is used to describe the turbulent-induced fading which covers the weak to strong turbulence regimes [6]. The effect of TX/RX aperture area upon the FSO system performance along with the comparison between single and multiple $(4 \times 4)$ aperture systems are also shown. This analysis will be very useful to scheme, predict and assess the performance of OFDM-FSO system under real weather conditions of Bangladesh and other places having similar subtropical monsoon climate.

\section{SYSTEM AND CHANNEL MODELING}

Figure 1 shows the basic configuration of OFDM-FSO system. In OFDM-FSO transmission system, QAM modulated RF signal is first converted into optical signal by the use of laser diode and then transmitted through the turbulent atmosphere by using FSO antenna. At the receiver end, the optical signal is received by FSO antenna and it is converted into RF signal using photo detector. In this transmission system, higher order modulation with less design complexity is used to transmit and receive the optical signals.

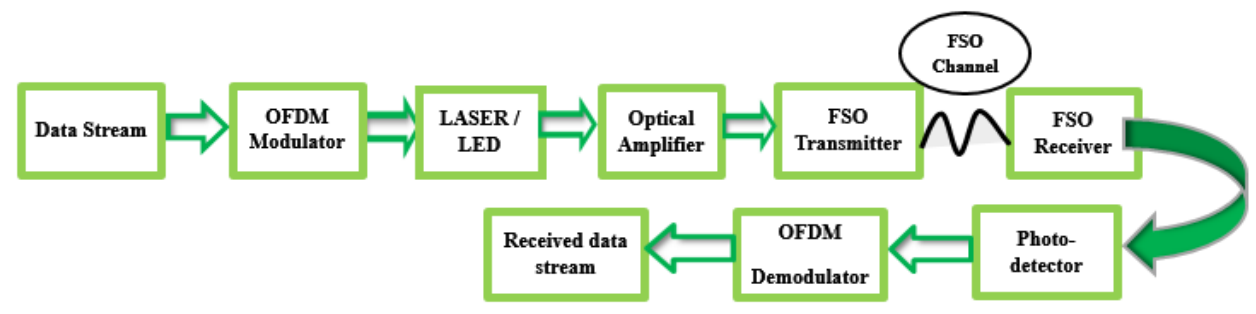

Figure 1. Basic architecture of OFDM-FSO system

\subsection{OFDM signal transmission and receiption over FSO link}

The basic concept behind OFDM technology is the division of a wideband frequency selective channel into multiple narrow band flat fading sub-channels in which the data bits can be transmitted in parallel through a large number of orthogonal subcarriers where the bandwidth of each subcarrier is much less than the channel coherence bandwidth [7].

Figure 2 shows the transceiver architecture of OFDM [8]. The input data is a serial stream of coded symbols. Serial to parallel converter converts the input serial data stream into a parallel stream of OFDM symbols. Higher order modulation such as 16-QAM is used as a mapping technique because it leads to increased spectral efficiency. After mapping the spectrum, an IFFT is used to find the corresponding time waveform. Training and Pilot symbols are also sent along with the transmitted data which helps in channel estimation. Cyclic prefix is also added at the starting of each symbol in order to avoid inter symbol interference and inter carrier interference [9]-[11]. Cubic interpolation techniques have been used to function as the digital to analog (DAC) converter. After the DAC, the parallel data is shifted back into the serial 
symbol stream. Later, this RF-OFDM signal is converted into an optical signal by using laser diode and transmitted through free space atmospheric channel.

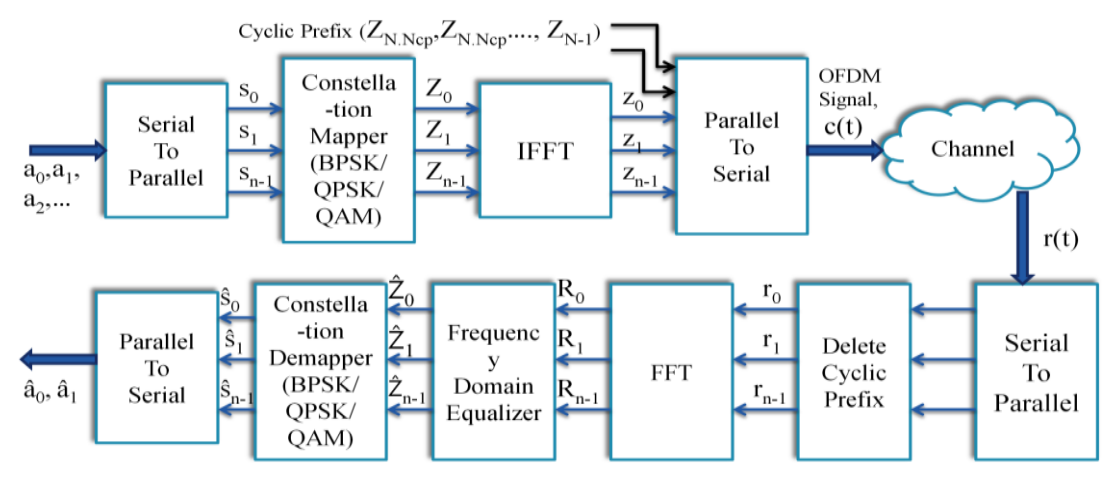

Figure 2. Transmitter and Receiver architecture of OFDM

The optical signal is received through FSO receiver and all reverse operations are carried out at the receiver side. With the help of photodetector optical signal is converted into RF electrical signal. The signal is sampled with analog to digital converter and guard retrieval is used for removal of guard bands. A single tap equalizer is applied to the subcarriers data to compensate for channel distortions. Fast Fourier Transform (FFT) is used to convert the signal back into the frequency domain. Finally, QAM decoder is used for demapping the symbols which are then converted from parallel to serial so that the further investigations can be carried out in terms of Bit Error Rate (BER) [12].

\subsection{FSO channel characteristics}

Free Space Optics (FSO) refers to the technology where modulated infrared or visible beams are transmitted through the air or atmosphere to obtain broadband communications. It consists of three subsystems: transmitter telescope, free space channel and receiver telescope [13]. The main objective of FSO is to receive a stronger signal that would result in higher link margin and greater link availability.

To evaluate the performance of FSO system, parameters are classified into two categories: Internal parameters and External parameters. Internal parameters are the system-specific design parameters such as: transmitter power, wavelength, transmitter and receiver aperture diameter, receiver sensitivity, beam divergence, transmitter and receiver losses. External parameters are the non-system-specific parameters (which can't be influenced by the designer) and deals with the climatology of the installation site, scintillation, atmospheric attenuation, window loss and pointing errors etc. [13].

The FSO link equation is given by Equation (1) [14]:

$$
P_{\text {received }}=P_{\text {transmitted }} \frac{d_{R}^{2}}{\left(d_{T}+\vartheta D\right)^{2}} 10^{-\tau \frac{D}{10}}
$$

Where, $d_{T}$ and $d_{R}$ are the transmitter and receiver aperture diameter respectively in $m, \vartheta$ is the beam divergence in mrad, $\mathrm{D}$ is the distance between optical transmitter and receiver in $\mathrm{km}$ and $\tau$ is the atmospheric attenuation coefficient $(\mathrm{dB} / \mathrm{km})$.

The geometric losses occur due to the spreading of the transmitted beam between the transmitter and receiver and can be approximated with the following formula [14]:

$$
\mathrm{G}_{\mathrm{L}}(d B)=20 \log \left[\frac{d_{R}}{d_{T}+\vartheta D}\right]
$$

\section{IMPACT OF ATMOSPHERE ON FSO SYSTEM IN PERSPECTIVE OF BANGLADESH}

The performance of FSO system is primarily dependent upon the climatology and the physical characteristics of its installation location. The primary factors affecting system performance include atmospheric attenuation causing scattering and absorption and atmospheric turbulence causing scintillation. 


\subsection{Atmospheric attenuation}

Atmospheric attenuation which degrades signal quality in a FSO system link, is generally dependent upon fog, low clouds, rain, dust, snow and various combinations of each. Fog typically dominates the signal aggravation. The several ways of signal degradation and attenuation by atmosphere includes absorption, scattering and scintillation. Being dependent on the local conditions and weather, all these effects vary with time. Atmospheric attenuation is given by the Beer's law [15] which is as follows:

$$
\tau=\exp ^{-\beta D}
$$

Where, $\tau$ is the atmospheric attenuation; $\mathrm{D}$ is the distance between transmitter and receiver $(\mathrm{km}) ; \beta$ is the total attenuation coefficient and given as:

$$
\beta=\beta_{\text {absorp }} \beta_{\text {scattering }}
$$

Here, $\beta_{\text {absorp }}$ is the molecular and aerosol absorption. The value of this parameter is considered as too small, so we can neglect it. $\beta_{\text {scattering }}$ is the molecular and aerosol scattering and it has a greater effect than absorption [15].

Scattering can be classified into three types: (a) Rayleigh scattering, (b) Mie scattering and (c) Non-selective scattering. Rayleigh scattering is negligible in the infrared waveband. Mie scattering occurs when the particles causing the scattering are equal to or larger than the wavelengths of radiation and it is the dominant scattering process in terrestrial FSO system. Non-selective scattering dominates when atmospheric particle size is much larger than the incoming radiation wavelength [16].

\subsubsection{Rain}

As the radius of raindrops $(100-1000 \mu \mathrm{m})$ is significantly larger than the wavelength of typical FSO systems, so scattering due to rainfall is called non-selective scattering. The rain scattering coefficient can be calculated using Stroke Law as shown in Equation (5) [17]:

$$
\beta_{\text {rain scatt }}=\pi r^{2} N_{r} Q_{\text {scat }}\left(\frac{r}{\lambda}\right)
$$

Here, $r$ is the radius of raindrop in $\mathrm{cm}, N_{r}$ is the rain drop distribution in $\mathrm{cm}^{-3}$ and $Q_{\text {scat }}$ is the scattering efficiency. The raindrop distribution $N_{r}$ can be calculated by using the following equation [17]:

$$
N_{r}=\frac{R}{1.33\left(\pi r^{3}\right) V_{L}}
$$

Where, $\mathrm{R}$ is the rainfall rate $(\mathrm{cm} / \mathrm{sec})$; and $V_{L}$ is the limiting speed of raindrop which is given by [17]:

$$
V_{L}=\frac{2 r^{2} \rho g}{9 \eta}
$$

Therefore, the rain attenuation can be calculated by using Beer's law as:

$$
\tau=\exp ^{-\beta_{\text {rain scatt }} D}
$$

Table 1. Raindrop Parameters for rain Attenuation Calculation

\begin{tabular}{cc}
\hline Parameter & Value \\
\hline Gravitational Constant, $g$ & $980 \mathrm{~cm} / \mathrm{s}^{2}$ \\
Viscosity of air, $\eta$ & $1.8 \times 10^{-4}(\mathrm{~g} / \mathrm{cm})$ \\
Radius of raindrop, $r$ & $0.05 \mathrm{~cm}$ \\
Water density, $\rho$ & $1 \mathrm{~g} / \mathrm{cm}^{3}$ \\
Wavelength, $\lambda$ & $1550 \mathrm{~nm}$ \\
$Q_{\text {scat }}$ & 2 \\
\hline
\end{tabular}

Using the values of the raindrop parameters given in Table 1 and data collected from the Meteorological Department of Bangladesh [18], we have calculated average rainfall rate for monsoon, pre-monsoon, postmonsoon period and their attenuation (shown in Table 2) utilizing the above mentioned formulas. 
Table 2. Rainfall Rate and Rain Attenuation Coefficient in Bangladesh

\begin{tabular}{ccc}
\hline Type & Rainfall Rate, $\mathrm{R}(\mathrm{cm} / \mathrm{sec})$ & Attenuation $(\mathrm{dB} / \mathrm{km})$ \\
\hline Pre-Monsoon & $4.7 \times 10^{-6}$ & 6.55 \\
Monsoon & $1.66 \times 10^{-5}$ & 23.12 \\
Post-Monsoon & $2.1345 \times 10^{-6}$ & 2.97 \\
\hline
\end{tabular}

\subsubsection{Fog}

Transmitted optical beams in free space are attenuated mostly by the fog droplets which is the key contributor to optical power/irradiance attenuation. The scattering coefficient $\beta_{a}$ due to fog can be expressed in terms of visibility and wavelength by the following expression [17]:

$$
\beta_{a}=\left(\frac{3.912}{V}\right)\left(\frac{\lambda}{550 \mathrm{~nm}}\right)^{-p}
$$

Where, $V$ is the visibility in $\mathrm{km}, \lambda$ is the incident laser beam wavelength in $\mathrm{nm}$ and $p$ is the size distribution of the scattering particles which typically varies from 0.7 to 1.6 corresponding to visibility conditions from poor to excellent. The particle size-related coefficient is found from the Kim model shown in Table 3:

Table 3. Scattering Particle Sizes at different Visibility Range (Kim Model)

\begin{tabular}{cc}
\hline Scattering Particle size, $p$ & Visibility Range $(\mathrm{km})$ \\
\hline 1.6 & $\mathrm{~V}>50$ \\
1.3 & $6<\mathrm{V}<50$ \\
$0.16 \mathrm{~V}+0.34$ & $1<\mathrm{V}<6$ \\
$\mathrm{~V}-0.5$ & $0.5<\mathrm{V}<1$ \\
0 & $\mathrm{~V}<0.5$ \\
\hline
\end{tabular}

The visibility at different foggy weather conditions of Bangladesh are collected from Bangladesh Meteorological Department [18] and for these visibility values the attenuation coefficients are calculated using the Kim Model. Table 4 shows the visibility and the calculated signal attenuation at different foggy weather conditions of Bangladesh.

Table 4. Signal Attenuation at different Foggy Weather Conditions of Bangladesh

\begin{tabular}{ccc}
\hline Weather Condition & Visibility $(\mathrm{km})$ & Attenuation $(\mathrm{dB} / \mathrm{km})$ \\
\hline Light Fog & 0.9 & 12.47 \\
Moderate Fog & 0.5 & 33.96 \\
Heavy Fog & 0.2 & 84.9 \\
\hline
\end{tabular}

\subsection{Atmospheric turbulence}

When signal is travelling through free space, atmospheric turbulence becomes an important factor results in random fluctuation of the refractive index along the optical propagation path. Inhomogeneity in the temperature, pressure and wind variations lead to change in the refractive index fluctuation. Atmospheric turbulence causes phase shifts of the propagating optical signals resulting in the wave front distortions and these distortions also cause intensity distortions, referred to as scintillation [19].

Refractive index structure parameter $\mathrm{C}_{n}^{2}$ determines the turbulence strength and clearly depends on the geographical location, altitude and time. A number of parametric models have been formulated to describe the $\mathrm{C}_{\mathrm{n}}{ }^{2}$ profile and a commonly used model to describe it is the Hufnagel-Valley model given as [20]:

$$
\begin{aligned}
C_{n}^{2}(h) & =0.0059\left(\frac{v}{27}\right)^{2}\left(10^{-5} h\right)^{10} \exp \left(-\frac{h}{1000}\right)+2.7 \times 10^{-16} \exp \left(-\frac{h}{1500}\right) \\
& +T_{0} \exp \left(-\frac{h}{100}\right)
\end{aligned}
$$

Where, $h$ is the altitude in meter, $v$ is the wind speed at high altitude in $\mathrm{m} / \mathrm{s}$ and $T_{o}$ is the turbulence strength at the ground level, $T_{o}=1.7 \times 10^{-14} \mathrm{~m}^{-2 / 3}$. 
The value of altitude and wind speed are collected from Bangladesh Meteorological Department [18] and using these values $\mathrm{C}_{\mathrm{n}}{ }^{2}$ is calculated which is in between $1.15 \times 10^{-14}$ to $1.7 \times 10^{-14} \mathrm{~m}^{-2 / 3}$. We consider the value of $\mathrm{C}_{\mathrm{n}}^{2}$ as $1.7 \times 10^{-14}$ throughout the whole simulation.

The gamma-gamma (GG) distribution is used to describe the PDF of the irradiance fluctuations and to model atmospheric fading [20]-[22]. In this case the probability of a given intensity $I_{R}$ of gamma-gamma scintillation model is [23]:

$$
P\left(I_{R}\right)=\frac{2(\alpha \beta)^{\frac{\alpha+\beta}{2}}}{\gamma(\alpha) \gamma(\beta)} I_{R}^{\frac{\alpha+\beta}{2}-1} K_{\alpha-\beta}\left(2 \sqrt{\alpha \beta I_{R}}\right) \quad ; \quad I_{R}>0
$$

Where, $\gamma(.$.$) is the Gamma function, K_{\alpha-\beta}(.$.$) is the modified Bessel function of the second kind, \alpha$ and $\beta$ are the variances of the small and large scale eddies respectively defined as [24]:

$$
\begin{aligned}
& \alpha=\left[\exp \left(\frac{0.49 \sigma_{R}^{2}}{\left(1+1.11 \sigma_{R}^{\frac{12}{5}}\right)^{\frac{7}{6}}}\right)-1\right]^{-1} \\
& \beta=\left[\exp \left(\frac{0.51 \sigma_{R}^{2}}{\left(1+0.69 \sigma_{R}^{\frac{12}{5}}\right)^{\frac{5}{6}}}\right)-1\right]^{-1}
\end{aligned}
$$

And the Rytov variance,

$$
\sigma_{\mathrm{R}}{ }^{2}=1.23 \mathrm{C}_{\mathrm{n}}^{2} \mathrm{k}^{7 / 6} \mathrm{D}^{11 / 6}
$$

Where, $k=2 \pi / \lambda$ is the wave number with $\lambda$ being the wavelength, and $D$ is the link distance.

Turbulence effect can be mitigated by using multiple transceivers. Multiple TX/RX combinations can raise the received power at the receiver. Thus the maximum achievable distance and BER can be improved [25]. Also, if the size of receiving aperture is larger than a spatial scale size that produces the irradiance fluctuations, the receiver will average the fluctuations over the aperture and the scintillation effect will be reduced [26].

\subsection{Optical loss, window loss and pointing error}

Optical loss depends on the characteristics of the equipment and lens quality. The value of this loss can be known from the component manufacturer. If FSO transceivers are installed behind windows within a building, an additional optical power loss occur due to the window glass attenuation. Coated windows show much greater attenuation than uncoated glass windows and its actual magnitude is typically wavelength dependent [14].

Pointing error is one of the key challenges faced by FSO links and additional power penalty is usually incurred due to imperfect alignment of the transmitter and receiver [14]. For shorter FSO links this might not be an issue, but for longer link distance pointing error can't be neglected.

\section{DESIGN PARAMETERS OF OFDM-FSO LINK}

An OFDM-FSO system is developed in which all possible atmospheric challenges for Bangladesh is considered under moderate atmospheric turbulence. The design parameters used for observing the performance of OFDM-FSO link in Bangladesh are shown in Table 5:

Table 5. Value of the Design Parameters for Performance Analysis

\begin{tabular}{cccc}
\hline Parameter Name & Value & Parameter Name & Value \\
\hline Bit Rate & $40 \mathrm{Gbps}$ & Dark Current & $10 \mathrm{nA}$ \\
Modulation Type & 16 QAM & Thermal Power & $100 \mathrm{e}^{-24} \mathrm{~W} / \mathrm{Hz}$ \\
Num. of OFDM Sub-Carriers & 256 & Amplifier Gain & $10 \mathrm{~dB}$ \\
Num. of used Sub-carriers & 80 & Noise Margin & $4 \mathrm{~dB}$ \\
\hline
\end{tabular}

Performance Investigation of OFDM-FSO System under Diverse Weather Conditions of ... (Maliha Sultana) 


\begin{tabular}{cccc}
\hline Parameter Name & Value & Parameter Name & Value \\
\hline Num. of Prefix Point & 50 & FSO Link Length, D & $0.1 \mathrm{~km}-4 \mathrm{~km}$ \\
Num. of Training Symbol & 10 & FSO Transmitter Aperture Diameter, $\mathrm{d}_{\mathrm{T}}$ & $0.025 \mathrm{~m}$ \\
Num. of Pilot Symbol & 9 & FSO Receiver Aperture Diameter, $\mathrm{d}_{\mathrm{R}}$ & $0.6 \mathrm{~m}$ \\
CW Laser Power & $20 \mathrm{dBm}$ & Beam Divergence, $\vartheta$ & $2 \mathrm{mrad}$ \\
Line width & $0.15 \mathrm{MHz}$ & Transmitter Loss & $1.8 \mathrm{~dB}$ \\
Channel Wavelength & $1550 \mathrm{~nm}$ & Receiver Loss & $1.8 \mathrm{~dB}$ \\
Photodetector Type & PIN & Additional Losses & $2 \mathrm{~dB}$ \\
Responsivity & $1 \mathrm{~A} / \mathrm{W}$ & Refractive structure Index for & $1.7 \times 10^{-14} \mathrm{~m}^{-2 / 3}$ \\
\hline
\end{tabular}

\section{RESULTS AND DISCUSSIONS}

The simulation results are divided into the following sections: BER Vs Link distance for different weather conditions of Bangladesh under moderate atmospheric turbulence at a fixed bit rate; Performance analysis of OFDM-FSO system by varying bit rate; Impact of moderate and strong atmospheric turbulence; Effect of aperture size on FSO performance along with the comparison between single and multiple aperture system.

\subsection{BER Vs Link distance for different weather conditions of Bangladesh under moderate turbulence}

In this paper, BER and received power are considered as performance parameters to characterize the OFDM-FSO system performance over fading atmospheric turbulence channels and atmospheric attenuations. By varying link distance, BER is recorded for different weather conditions of Bangladesh at 40 Gbps data rate. During these simulations, all possible challenges are considered under moderate atmospheric turbulence.

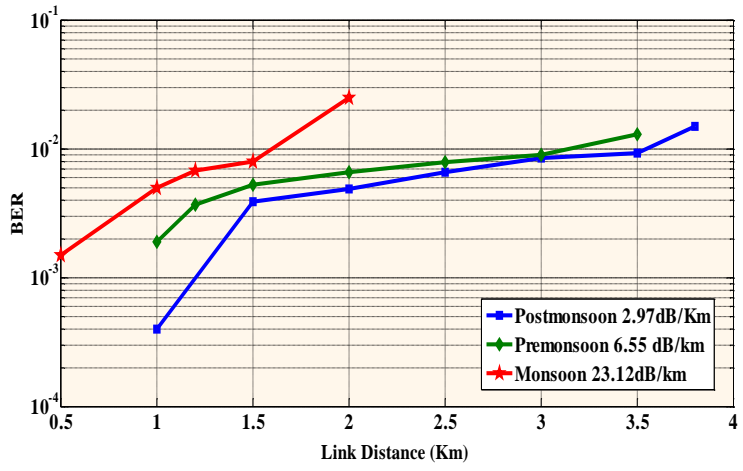

Figure 3. BER Vs Link distance at rainy weather

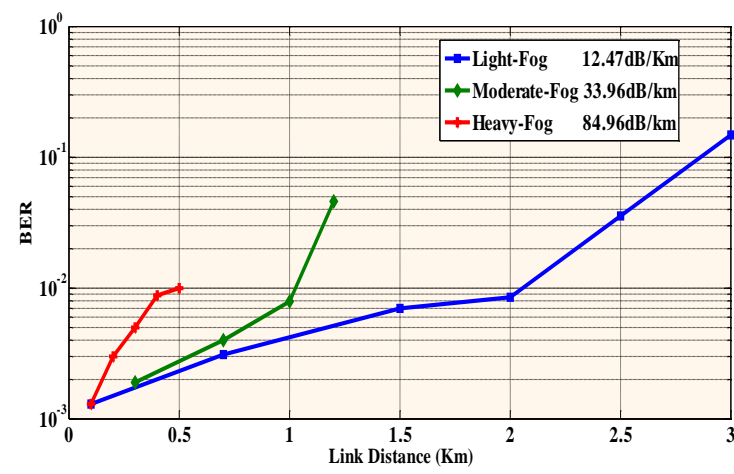

Figure 4. BER Vs Link distance at foggy weather

Figure 3 and Figure 4 depict the BER Vs link distance for rain and fog respectively showing the effect of attenuation on BER. With the increase of link distance, the transmitted signal strength gets attenuated due to different weather conditions. Consequently, the BER also degrades.

From Figure 3, we can conclude that the maximum attainable link distance during pre-monsoon, monsoon and post-monsoon periods of Bangladesh are $3 \mathrm{Km}, 1.5 \mathrm{Km}$ and $3.5 \mathrm{Km}$ respectively to maintain a BER performance in the order of $10^{-3}$ at $40 \mathrm{Gbps}$ bit rate.

Similarly from Figure 4, it can be concluded that the maximum attainable link distance during light fog, moderate fog and heavy fog conditions are $2 \mathrm{Km}, 1 \mathrm{Km}$ and $0.4 \mathrm{Km}$ respectively maintaining a BER at the order of $10^{-3}$. Table 6 summarizes these results. After this link margin, transmitted data gets severely attenuated with increased BER which is difficult to recover.

Table 6. Maximum Link range For Rain and Fog (from Figure 3 and Figure 4)

\begin{tabular}{|c|c|c|c|c|c|}
\hline Weather & $\begin{array}{c}\text { Attenuation } \\
(\mathrm{dB} / \mathrm{Km})\end{array}$ & Bit Rate & $\begin{array}{c}\text { Link Distance } \\
(\mathrm{Km})\end{array}$ & BER & $\begin{array}{c}P_{\text {Receieved }} \\
(\mathrm{dBm})\end{array}$ \\
\hline Pre-Monsoon (Moderate rain) & 6.55 & & 3 & $9 \times 10^{-3}$ & -23.28 \\
\hline Monsoon (Heavy rain) & 23.12 & 40Gbps & 1.5 & $8 \times 10^{-3}$ & -32.28 \\
\hline Post-Monsoon (Light Rain) & 2.97 & & 3.5 & $9.3 \times 10^{-3}$ & -15.30 \\
\hline Light Fog & 12.47 & 40Gbps & 2 & $8.5 \times 10^{-3}$ & -25.043 \\
\hline
\end{tabular}




\begin{tabular}{|c|c|c|c|c|c|}
\hline Weather & $\begin{array}{c}\text { Attenuation } \\
(\mathrm{dB} / \mathrm{Km})\end{array}$ & $\begin{array}{c}\text { Bit } \\
\text { Rate }\end{array}$ & $\begin{array}{c}\text { Link Distance } \\
(\mathrm{Km})\end{array}$ & BER & $\begin{array}{c}P_{\text {Receieved }} \\
(\mathrm{dBm})\end{array}$ \\
\hline Moderate Fog & 33.96 & & 1 & $7.9 \times 10^{-3}$ & -28.096 \\
\hline Heavy Fog & 84.9 & & 0.4 & $8.8 \times 10^{-3}$ & -20.303 \\
\hline
\end{tabular}

\subsection{BER vs bit rate for different weather conditions of Bangladesh under moderate turbulence}

Figure 5 shows the BER performance of OFDM-FSO transmission system as a function of bit rate for heavy rain and moderate fog under moderate atmospheric turbulence. It is clear that the performance starts to degrade with the increase of bit rate. Also it can be seen that at the range of $1 \mathrm{Km}, 45 \mathrm{Gbps}$ can be sent at a BER of $7 \times 10^{-3}$ during heavy rain whereas 43 Gbps can be sent at a similar BER under moderate fog but within the range of $0.7 \mathrm{Km}$. Beyond this bit rate, transmitted signal gets attenuated with a BER greater than $10^{-3}$.

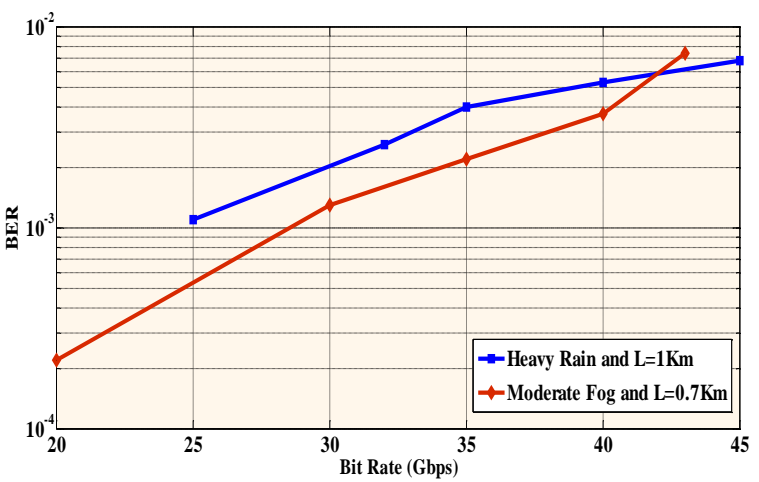

Figure 5. BER vs bit rate for heavy rain and moderate fog

\subsection{Impact of moderate and strong atmospheric turbulence}

The FSO link performance is affected by the scintillation due to different types of atmospheric turbulence. Table 7 illustrates the effect of moderate and strong turbulence on OFDM-FSO system performance under heavy rain and moderate fog weather conditions of Bangladesh. It is seen that the effect of turbulence deteriorates the link performance. For moderate turbulence, BER remains in the order of $10^{-3}$ whereas it becomes $10^{-2}$ under the influence of strong turbulence. Therefore, if we want to keep the BER of strong turbulence in the order of $10^{-3}$, we have to compromise the data rate and/or link distance.

Table 7. Effect of Turbulence on OFDM-FSO Performance

\begin{tabular}{cccc}
\hline Turbulence Type, $\mathrm{C}_{\mathrm{n}}{ }^{2}$ & $\begin{array}{c}\text { Attenuation } \\
(\mathrm{dB} / \mathrm{km})\end{array}$ & Link Distance $(\mathrm{Km})$ & BER \\
\hline & $23.12($ Heavy rain) & 1.5 & $8.0 \times 10^{-3}$ \\
Moderate $\left(1.7 \mathrm{e}^{-14} \mathrm{~m}^{-2 / 3}\right)$ & 33.96 (Moderate Fog) & 1 & $7.9 \times 10^{-3}$ \\
& 23.12 (Heavy rain) & 1.5 & $3.3 \times 10^{-2}$ \\
Strong $\left(4.58 \mathrm{e}^{-13} \mathrm{~m}^{-2 / 3}\right)$ & 33.96 (Moderate Fog) & 1 & $3.0 \times 10^{-2}$ \\
\hline
\end{tabular}

\subsection{Effect of aperture size variation on OFDM-FSO performance along with comparison between single and multiple aperture systems}

In this section, different TX/RX aperture diameter values are used for the analysis of system performance considering all possible losses under moderate turbulence. Table 8 shows that the BER is minimum when $\mathrm{d}_{\mathrm{T}}=2.5 \mathrm{~cm}$ and $\mathrm{d}_{\mathrm{R}}=60 \mathrm{~cm}$ with $20 \mathrm{dBm}$ power at $40 \mathrm{Gbps}$ data rate and $0.7 \mathrm{~km}$ link range in moderate foggy weather.

From Table 8, it can be said that the larger the receiver aperture area, the smaller the Bit Error Rate. It also elicits that there is a huge BER improvement while using $4 \times 4$ aperture system compared to the corresponding single aperture system. 
Table 8. BER Performance of Single and 4×4 Multiple Aperture System

\begin{tabular}{cccc}
\hline Type & $\mathrm{d}_{\mathrm{T}}(\mathrm{cm})$ & $\mathrm{d}_{\mathrm{R}}(\mathrm{cm})$ & $\mathrm{BER}$ \\
\hline & 2.5 & 25 & $3.3 \times 10^{-2}$ \\
$4 \times 4$ & 2.5 & 40 & $1.0 \times 10^{-2}$ \\
Multiple & 2.5 & 60 & $4.4 \times 10^{-3}$ \\
Beam & 5 & 25 & $5.0 \times 10^{-2}$ \\
& 5 & 40 & $1.1 \times 10^{-2}$ \\
& 5 & 60 & $7.0 \times 10^{-3}$ \\
& 8 & 25 & $4.7 \times 10^{-2}$ \\
& 8 & 40 & $1.9 \times 10^{-2}$ \\
Single Beam & 8 & 60 & $9.0 \times 10^{-3}$ \\
& 2.5 & 60 & $2.0 \times 10^{-1}$ \\
\hline
\end{tabular}

\section{CONCLUSION}

This paper investigates the OFDM-FSO system performance in Gamma-Gamma fading under diverse weather conditions of Bangladesh. The data from Bangladesh Meteorological Department are used for calculating the refractive index structure parameter and atmospheric attenuation coefficient for different weather conditions. The calculated attenuation coefficient, misalignment and other losses are taken into account during this investigation considering moderate atmospheric turbulence. BER is used as performance parameter and link distance is varied from $0.1 \mathrm{~km}-4 \mathrm{~km}$ at $40 \mathrm{Gbps}$ data rate to observe the system performance. The investigation provides the maximum attainable link ranges keeping a BER in the order of $10^{-3}$ at different weather conditions. Heavy fog is found to be the worst weather condition for OFDM-FSO system where the maximum attainable link range is found as only $0.4 \mathrm{~km}$ at a BER of $8.8 \times 10^{-3}$, whereas for light rain weather condition the maximum attainable link range is $3.5 \mathrm{~km}$ at a BER of $9.3 \times 10^{-3}$. The effect of transmitter and receiver aperture size variation on OFDM-FSO performance is also investigated, which shows that if the receiver aperture area is increased the BER is reduced. The comparison between single and multiple aperture system illustrates that there is a huge BER improvement while using $4 \times 4$ aperture system compared to the corresponding single aperture system. The outcomes of this paper would help in designing FSO links over longer distances for Bangladesh and promote the usage of this technology beyond the last mile solution in near future.

\section{ACKNOWLEDGEMENTS}

The authors would like to thank Md. Jahedul Islam, Department of Electrical and Electronic Engineering, Khulna University of Engineering and Technology for his technical advice and support.

\section{REFERENCES}

[1] Hemani Kaushaland, et al., "Optical Communicaton in space: Challenges and Mitigation Techniques", IEEE Communications Surveys and Tutorials; vol. 19, no. 1, pp. 57-96, 2017 Jan 1.

[2] Majumdar AK. , "Advanced Free Space Optics (FSO): A Systems Approach”, Springer; 2014 Sep 10.

[3] Suyoto S, Iskandar I, Sugihartono S, Kurniawan A, "Improved Timing Estimation Using Iterative Normalization Technique for OFDM Systems", International Journal of Electrical and Computer Engineering (IJECE), vol. 7, no. 2, pp. 905-911, 2017 Apr 1.

[4] Chuang J, Sollenberger N., "Beyond 3G: Wideband Wireless Data Access based on OFDM and Dynamic Packet Assignment”, IEEE Communications Magazine, vol. 38, no. 7, pp. 78-87, 2000 Jul.

[5] Jain M, Roja MM, "Comparison of OFDM with CDMA System in Wireless Telecommunication for Multipath Delay Spread", In Internet, 2005, The First IEEE and IFIP International Conference in Central Asia on 2005 Sep 26 (pp. 5-pp).

[6] Khalighi MA, et al., "Fading Reduction by Aperture Averaging and Spatial Diversity in Optical Wireless Systems", Journal of Optical communications and networking, vol. 1, no. 6, pp. 580-593, 2009 Nov 1.

[7] Jalili A, Amirani MC, Kordan SB, "A Blind Carrier Frequency Offset Estimation Scheme for OFDM Systems via Hybrid-ICA Algorithm”, International Journal of Electrical and Computer Engineering (IJECE), vol. 4, no. 5, p. 790, 2014 Oct 1.

[8] Oltean M., "An Introduction to Orthogonal Frequency Division Multiplexing”, Analele Universitatii Oradea, 2004, Fascicola Electrotehnica, Sectiunea Electronica (2004): 180-185.

[9] Armstrong J, “OFDM for Optical Communications", Journal of Lightwave Technology, vol. 27, no. 3, pp. 189-204, 2009 Feb 1.

[10] Dhawan D, Gupta N, "Performance Analysis of Post Compensated Long Haul High Speed Coherent Optical OFDM System", International Journal of Electrical and Computer Engineering (IJECE), vol. 7, no. 1, pp. 160-168, 2017 Feb 1. 
[11] Yadav SP, Bera SC, "PAPR Reduction for Improved Efficiency of OFDM Modulation for Next Generation Communication Systems", International Journal of Electrical and Computer Engineering (IJECE), vol. 6, no. 5, p. 2310, 2016 Oct 1 .

[12] Sukhpal Singh, Harmanjot Singh, "Review Paper on OFDM-Concepts and Applications", International Journal of Engineering Development and Research, vol. 3, no. 3, 2015.

[13] Alsemmeari RA, et al., "Free Space Optics Vs Radio Frequency Wireless Communication", International Journal of Information Technology and Computer Science (IJITCS), vol. 8, no. 9, p. 1, 2016 Sep 8.

[14] Bloom S, et al., "Understanding the Performance of free-space Optics", Journal of Optical Networking, vol. 2, no. 6, pp. 178-200, 2003 Jun 6.

[15] Kartik Ramesh Patel, et al., "Free Space Optics (FSO) - Past, Present, Future and Mathematical Models of Atmospheric Turbulence for FSO Link Budget Analysis", International Journal on Recent and Innovation Trends in Computing and Communication, pp. 149-154, 2016 Jan.

[16] Alkholidi AG, Altowij KS, "Free Space Optical Communications-Theory and Practices", In Contemporary Issues in Wireless Communications 2014, InTech.

[17] Fadhil HA, et al., "Optimization of free space Optics Parameters: An Optimum Solution for bad weather conditions", Optik-International Journal for Light and Electron Optics, vol. 124, no. 19, pp. 3969-3973, 2013 Oct 31

[18] Meteorological Department of Bangladesh, http://www.bmd.gov.bd/ [31 January, 2018].

[19] Saleem Z, Khan N, Ishaq W, Altaf M, "Free Space Optical (FSO) link Design under diverse weather conditions", WSEAS Transactions on Electronics, vol. 3, no. 4, pp. 225-232, 2006 Apr.

[20] Andrews LC, Phillips RL, Hopen CY, "Laser Beam Scintillation with Applications”, vol. 99, SPIE press; 2001.

[21] Andrews LC, Phillips RL, "Laser Beam Propagation through random Media", vol. 152, Bellingham, WA: SPIE press; 2005 Sep 16.

[22] Farid AA, Hranilovic S, "Outage Capacity Optimization for free-space Optical Links with Pointing Errors", Journal of Lightwave Technology, vol. 25, no. 7, pp. 1702-1710, 2007 Jul 1.

[23] Kumar P, Srivastava A., "Enhanced performance of FSO link using OFDM and comparison with traditional TDMFSO link", In Broadband and Photonics Conference (IBP), 2015 IEEE International, 2015 Apr 23 (pp. 65-70). IEEE.

[24] Ghassemlooy Z, Popoola WO, Leitgeb E, "Free-Space Optical Communication using Subcarrier Modulation in Gamma-Gamma Atmospheric Turbulence”, In Transparent Optical Networks, 2007. ICTON'07. 9th International Conference on 2007 Jul 1 (vol. 3, pp. 156-160). IEEE.

[25] Noor NH, et al., "Performance Analysis of a free space Optics Link with Multiple Transmitters/receivers", IIUM Engineering Journal, vol. 13, no. 1, 2012 Apr 20.

[26] Churnside JH., "Aperture Averaging of Optical Scintillations in the Turbulent Atmosphere", Applied Optics, 1991 May 20, vol. 30, no. 15, pp. 1982-1994. 